\title{
Synchronization of Uncertain Fractional-Order Hyperchaotic Systems via Unidirectional Linear Error Feedback Coupling Scheme
}

\author{
Suwat Kuntanapreeda \\ Department of Mechanical and Aerospace Engineering, Faculty of Engineering, \\ King Mongkut's University of Technology North Bangkok, Bangkok 10800, Thailand \\ Correspondence should be addressed to Suwat Kuntanapreeda; suwat@kmutnb.ac.th
}

Received 23 May 2013; Revised 16 August 2013; Accepted 19 August 2013

Academic Editor: Qingdu Li

Copyright (C) 2013 Suwat Kuntanapreeda. This is an open access article distributed under the Creative Commons Attribution License, which permits unrestricted use, distribution, and reproduction in any medium, provided the original work is properly cited.

A simple method for synchronization of uncertain fractional-order hyperchaotic systems is proposed in this paper. The method makes use of a unidirectional linear coupling approach due to its simple configuration and ease of implementation. To determine the coupling parameters, the synchronization error dynamics is first formulated as a fractional-order linear interval system. Then, the parameters are obtained by solving a linear matrix inequality (LMI) stability condition for stabilization of fractional-order linear interval systems. Thanks to the existence of an LMI solution, the convergence of the synchronization errors is guaranteed. The effectiveness of the proposed method is numerically illustrated by the uncertain fractional-order hyperchaotic Lorenz system.

\section{Introduction}

Chaos synchronization has attracted great attention due to its superior potential applications, for example, in communication and optics. It has been studied since the pioneering work of Pecora and Carroll [1] was published. Currently, studies of chaos control and synchronization have more focus on hyperchaotic systems because of their rich chaos behaviors.

Although the notion of fractional calculus dates from the 17th century [2], its practical applications have just recently been investigated. In recent year, it has been demonstrated that fractional-order systems could behave chaotically or hyperchaotically. Examples of such systems include the fractional-order Chua system [3], the fractional-order Chen system [4], the fractional-order hyperchaotic Lorenz system [5], and the fractional-order hyperchaotic Chen system [6].

Nowadays, whereas chaos synchronization of conventional integer-order chaotic and hyperchaotic systems has been extensively studied, chaos synchronization of fractionalorder chaotic and hyperchaotic systems is still considered as a challenging research topic. Examples of existing methods for chaos synchronization of fractional-order chaotic and hyperchaotic systems are an active control method $[7,8]$, a sliding-mode control method $[9,10]$, a robust control method [11, 12], an adaptive control method [13], and a tracking control-based method $[14,15]$. The sliding-mode control, robust control, and adaptive control are commonly used methods to cope with the nonlinear systems that have some uncertain parameters.

This paper extends the robust control approach presented in [12] to synchronization of uncertain fractionalorder hyperchaotic systems. A unidirectional linear error feedback coupling scheme is adopted here due to its simple configuration and ease of implementation in real systems. The method makes use of an LMI stability condition of fractionalorder interval linear systems. An obtained solution to the LMI condition yields the values of the coupling parameters and guarantees the stability of the synchronization error dynamics.

The rest of the paper is organized as follows. In the next section, some preliminaries are presented. The main results are given in Section 3. In Section 4, the uncertain fractionalorder hyperchaotic Lorenz system that is used as an illustrative example is described. Synchronization of the system 
is also given in this section. Section 5 presents numerical results that illustrate the effectiveness of the method. Finally, conclusions are drawn in the last section.

\section{Preliminaries}

There are several definitions of fractional derivative [2]. The frequently found definitions for fractional derivatives are Riemann-Liouville, Grünwald-Letnikov, and Caputo definitions. In this paper, the Caputo definition is adopted.

A fractional-order LTI interval system with no input is described as [16]

$$
D^{q} x=\breve{A} x
$$

where $x=\left[x_{1}, x_{2}, \ldots, x_{n}\right]^{T}$ is the state vector, $q$ is the fractional commensurate order, and the system matrix $\breve{A}$ is interval uncertain satisfying

$$
\breve{A} \in\left[A^{l}, A^{u}\right]=\left\{\left[a_{i j}\right]: a_{i j}^{l} \leq a_{i j} \leq a_{i j}^{u}, 1 \leq i, j \leq n\right\} .
$$

The following notations are used in the following lemma:

$$
\begin{aligned}
& A_{0}=\frac{1}{2}\left(A^{l}+A^{u}\right) \\
& \Delta A=\frac{1}{2}\left(A^{l}-A^{u}\right)=\left[\gamma_{i j}\right],
\end{aligned}
$$

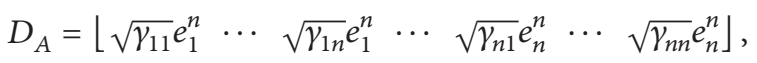

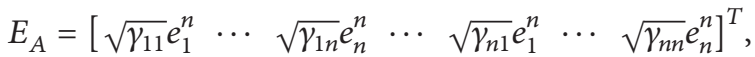

where $e_{i}^{p}$ is the $p$-column vector with the $i$ th element being 1 and all the other being 0 .

Lemma 1 (see [16]). The interval system (1) with $0<q<1$ is robustly asymptotically stable if and only if there exist two $n \times n$ real symmetric positive-definite matrices $P_{k 1}(k=1,2)$, two $n \times n$ skew-symmetric matrices $P_{k 2}(k=1,2)$, and four real positive scalars $\varepsilon_{i j}(i, j=1,2)$ such that

$$
\begin{gathered}
\Omega=\left[\begin{array}{ll}
\Omega_{11} & \Omega_{12} \\
\Omega_{12}^{T} & \Omega_{22}
\end{array}\right]<0, \\
{\left[\begin{array}{cc}
P_{11} & P_{12} \\
-P_{12} & P_{11}
\end{array}\right]>0, \quad\left[\begin{array}{cc}
P_{21} & P_{22} \\
-P_{22} & P_{21}
\end{array}\right]>0,}
\end{gathered}
$$

where

$$
\Omega_{11}=\sum_{i=1}^{2} \sum_{j=1}^{2}\left\{\operatorname{Sym}\left\{\Theta_{i j} \otimes\left(A_{0} P_{i j}\right)\right\}+\varepsilon_{i j}\left(I_{2} \otimes D_{A} D_{A}^{T}\right)\right\},
$$

$\Omega_{12}$

$$
\begin{aligned}
& =\left[I_{2} \otimes\left(E_{A} P_{11}\right)^{T} I_{2} \otimes\left(E_{A} P_{12}\right)^{T} I_{2} \otimes\left(E_{A} P_{21}\right)^{T} I_{2} \otimes\left(E_{A} P_{22}\right)^{T}\right], \\
& \Omega_{22}=-\operatorname{diag}\left(\varepsilon_{11}, \varepsilon_{12}, \varepsilon_{21}, \varepsilon_{22}\right) \otimes I_{2 n}, \\
& \Theta_{11}=\left[\begin{array}{cc}
\sin (\theta) & -\cos (\theta) \\
\cos (\theta) & \sin (\theta)
\end{array}\right], \quad \theta=q \frac{\pi}{2}, \\
& \Theta_{12}=\left[\begin{array}{cc}
\cos (\theta) & \sin (\theta) \\
-\sin (\theta) & \cos (\theta)
\end{array}\right], \quad \Theta_{21}=\left[\begin{array}{cc}
\sin (\theta) & \cos (\theta) \\
-\cos (\theta) & \sin (\theta)
\end{array}\right], \\
& \Theta_{22}=\left[\begin{array}{cc}
-\cos (\theta) & \sin (\theta) \\
-\sin (\theta) & -\cos (\theta)
\end{array}\right], \quad \operatorname{Sym}\{Z\}=Z^{T}+Z,
\end{aligned}
$$

and $\otimes$ is the Kronecker product.

\section{Main Results}

Consider a fractional-order hyperchaotic system of order $q$ $(0<q<1)$ described by

$$
D^{q} \mathscr{X}=F(\mathscr{X})
$$

where $\mathscr{X}$ is the state vector. Two systems in synchronization are called the master system and the slave system, respectively. By utilizing a unidirectional linear error feedback coupling scheme, the master system and the slave system are constructed, respectively, as

$$
\begin{gathered}
D^{q} \mathscr{X}_{m}=F\left(\mathscr{X}_{m}\right), \\
D^{q} \mathscr{X}_{s}=F\left(\mathscr{X}_{s}\right)-B\left(\mathscr{X}_{s}-\mathscr{X}_{m}\right),
\end{gathered}
$$

where the subscripts $m$ and $s$ stand for the master and the slave, respectively, and $B=\operatorname{diag}\left(\beta_{i}\right)(i=1,2, \ldots, n)$ is the coupling matrix, $\beta_{i}(i=1,2, \ldots, n)$ are called the coupling parameters that will be designed.

By defining the synchronization error vector as $\mathscr{E}=\mathscr{X}_{s}-$ $\mathscr{X}_{m}$ and using (7), the synchronization error system can be written as

$$
D^{q} \mathscr{E}=G\left(\mathscr{E}, \mathscr{X}_{s}, \mathscr{X}_{m}\right)-B \mathscr{E},
$$

where $G\left(\mathscr{E}, \mathscr{X}_{s}, \mathscr{X}_{m}\right)=F\left(\mathscr{X}_{s}\right)-F\left(\mathscr{X}_{m}\right)$. Next, assume that the error system (8) can be expressed in a linear interval system as

$$
D^{q} \mathscr{E}=\breve{A} \mathscr{E}-B \mathscr{E}=\breve{A}_{c} \mathscr{E},
$$

where $\breve{A}$ and $\breve{A}_{c}=\breve{A}-B$ are the interval matrices of the uncoupled and coupled systems, respectively.

Theorem 2. The linear interval system (9) with $0<q<1$ is asymptotically stable if there exist an $n \times n$ real diagonal 
positive-definite matrix $Q$, an $n \times n$ diagonal matrix $\Lambda$, and four real positive scalars $\alpha_{i}(i=1,2,3,4)$ such that

$$
\Psi=\left[\begin{array}{ll}
\Psi_{11} & \Psi_{12} \\
\Psi_{12}^{T} & \Psi_{22}
\end{array}\right]<0
$$

where

$$
\begin{aligned}
& \Psi_{11}=\sum_{i=1}^{2} \operatorname{Sym}\left\{\Theta_{i 1} \otimes\left(A_{0} Q-\Lambda\right)\right\} \\
& +\sum_{i=1}^{4} \alpha_{i}\left(I_{2} \otimes D_{A} D_{A}^{T}\right), \\
& \Psi_{12}=\left[\begin{array}{llll}
I_{2} \otimes\left(E_{A} Q\right)^{T} & 0_{2 n} & I_{2} \otimes\left(E_{A} Q\right)^{T} & 0_{2 n}
\end{array}\right], \\
& \Psi_{22}=-\operatorname{diag}\left(\alpha_{1}, \alpha_{2}, \alpha_{3}, \alpha_{4}\right) \otimes I_{2 n}
\end{aligned}
$$

and $A_{0}, D_{A}, E_{A}$ are the matrices that associate with $\breve{A}$. Moreover, the coupling matrix is given by

$$
B=\Lambda Q^{-1} \text {. }
$$

Proof. Suppose that there exist an $n \times n$ real diagonal positivedefinite matrix $Q$, an $n \times n$ diagonal matrix $\Lambda$, and four real positive scalars $\alpha_{i}(i=1,2,3,4)$ such that (10) and (12) hold. By introducing $Q=P_{11}=P_{21}, \Lambda=B Q$, and $\alpha_{1}=\varepsilon_{11}, \alpha_{2}=$ $\varepsilon_{12}, \alpha_{3}=\varepsilon_{21}$, and $\alpha_{4}=\varepsilon_{22}$ into (11), it results

$$
\Psi_{11}=\sum_{i=1}^{2} \sum_{j=1}^{2}\left\{\operatorname{Sym}\left\{\Theta_{i j} \otimes\left(A_{0}-B\right) P_{i j}\right\}+\varepsilon_{i j}\left(I_{2} \otimes D_{A} D_{A}^{T}\right)\right\},
$$

$\Psi_{12}$

$$
=\left[I_{2} \otimes\left(E_{A} P_{11}\right)^{T} \quad I_{2} \otimes\left(E_{A} P_{12}\right)^{T} \quad I_{2} \otimes\left(E_{A} P_{21}\right)^{T} \quad I_{2} \otimes\left(E_{A} P_{22}\right)^{T}\right],
$$

$$
\Psi_{22}=-\operatorname{diag}\left(\varepsilon_{11}, \varepsilon_{12}, \varepsilon_{21}, \varepsilon_{22}\right) \otimes I_{2 n}
$$

where $P_{12}=P_{22}=0$. Note that

$$
\begin{aligned}
& {\left[\begin{array}{cc}
P_{11} & P_{12} \\
-P_{12} & P_{11}
\end{array}\right]=\left[\begin{array}{cc}
Q & 0 \\
0 & Q
\end{array}\right]>0,} \\
& {\left[\begin{array}{cc}
P_{21} & P_{22} \\
-P_{22} & P_{21}
\end{array}\right]=\left[\begin{array}{cc}
Q & 0 \\
0 & Q
\end{array}\right]>0 .}
\end{aligned}
$$

Next, consider

$$
\breve{A}_{c}=\breve{A}-B \text {. }
$$

Since $B$ is certain,

$$
\begin{gathered}
\breve{A}_{c} \in\left[A_{c}^{l}, A_{c}^{u}\right]=\left[A^{l}-B, A^{u}-B\right], \\
A_{c 0}=\frac{1}{2}\left(A_{c}^{l}+A_{c}^{u}\right)=A_{0}-B, \\
\Delta A_{c}=\frac{1}{2}\left(A_{c}^{l}-A_{c}^{u}\right)=\Delta A .
\end{gathered}
$$

Using (19),

$$
\Psi_{11}=\sum_{i=1}^{2} \sum_{j=1}^{2}\left\{\operatorname{Sym}\left\{\Theta_{i j} \otimes A_{c 0} P_{i j}\right\}+\varepsilon_{i j}\left(I_{2} \otimes D_{A} D_{A}^{T}\right)\right\} .
$$

From (10), (14), (15), (16), and (21) and Lemma 1, it can be concluded that the system (9) is asymptotically stable. This ends the proof.

\section{Chaos Synchronization}

The uncertain fractional-order hyperchaotic Lorenz system considered here as the illustrative example is given by

$$
\begin{gathered}
D^{\alpha} x=(a+\Delta a)(y-x)+w, \\
D^{\alpha} y=(c+\Delta c) x-y-x z, \\
D^{\alpha} z=-(b+\Delta b) z+x y, \\
D^{\alpha} w=-y z+(r+\Delta r) w,
\end{gathered}
$$

where $a>0, b>0, c>0$, and $r$ are the nominal parameters of the system, $\Delta a, \Delta b, \Delta c$, and $\Delta r$ are additive uncertainties whose magnitudes are bounded by $\sigma_{a}, \sigma_{b}, \sigma_{c}$, and $\sigma_{r}$, respectively, and $0<\alpha<1$ is the fractional commensurate order. When $a=10, b=8 / 3, c=28$, $r=-1$, and $\alpha=0.98$, the nominal system of (22) has a chaotic attractor as shown in Figure 1.

From (22), the master and slave systems can be expressed as

$$
\begin{gathered}
D^{\alpha} x_{m}=(a+\Delta a)\left(y_{m}-x_{m}\right)+w_{m}, \\
D^{\alpha} y_{m}=(c+\Delta c) x_{m}-y_{m}-x z_{m}, \\
D^{\alpha} z_{m}=-(b+\Delta b) z_{m}+x_{m} y_{m}, \\
D^{\alpha} w_{m}=-y_{m} z_{m}+(r+\Delta r) w_{m}, \\
D^{\alpha} x_{s}=(a+\Delta a)\left(y_{s}-x_{s}\right)+w_{s}-\beta_{1}\left(x_{s}-x_{m}\right), \\
D^{\alpha} y_{s}=(c+\Delta c) x_{s}-y_{s}-x_{s} z_{s}-\beta_{2}\left(y_{s}-y_{m}\right), \\
D^{\alpha} z_{s}=-(b+\Delta b) z_{s}+x_{s} y_{s}-\beta_{3}\left(z_{s}-z_{m}\right), \\
D^{\alpha} w_{s}=-y_{s} z_{s}+(r+\Delta r) w_{s}-\beta_{4}\left(w_{s}-w_{m}\right),
\end{gathered}
$$

where the lower scripts $m$ and $s$ stand for the master and slave, respectively, and $\beta_{i}(i=1,2,3,4)$ are the coupling parameters that are designed such that the two systems are synchronized.

By defining the synchronization errors as $e_{1}=x_{s}-x_{m}$, $e_{2}=y_{s}-y_{m}, e_{3}=z_{s}-z_{m}$, and $e_{4}=w_{s}-w_{m}$. Using (23), the error system can be expressed as

$$
\begin{gathered}
D^{\alpha} e_{1}=-(a+\Delta a) e_{1}+(a+\Delta a) e_{2}+e_{4}-\beta_{1} e_{1}, \\
D^{\alpha} e_{2}=\left(c+\Delta c-z_{s}\right) e_{1}-e_{2}-x_{m} e_{3}-\beta_{2} e_{2}, \\
D^{\alpha} e_{3}=y_{m} e_{1}+y_{s} e_{2}-(b+\Delta b) e_{3}-\beta_{3} e_{3}, \\
D^{\alpha} e_{4}=-z_{m} e_{2}-y_{s} e_{3}+(r+\Delta r) e_{4}-\beta_{4} e_{4} .
\end{gathered}
$$



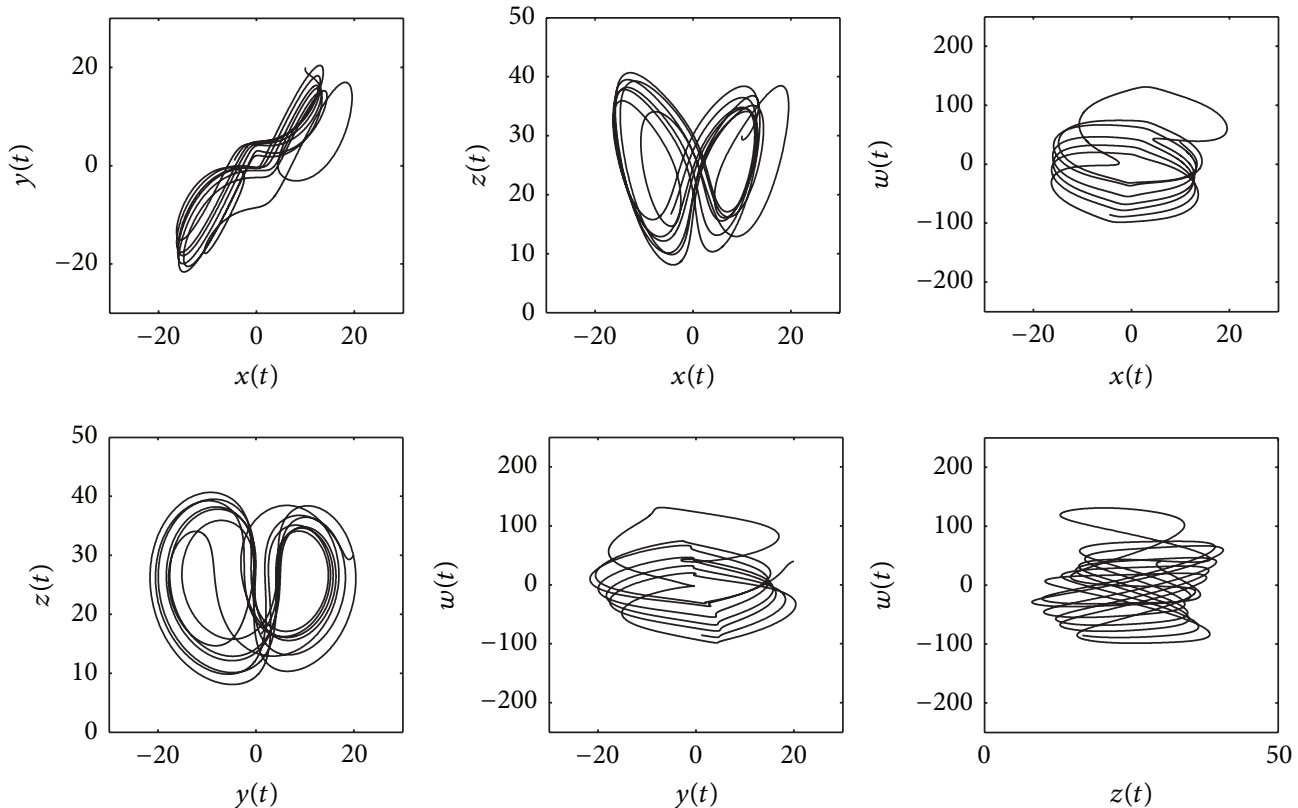

Figure 1: Phase portrait of the fractional-order hyperchaotic Lorenz system.

Due to the attractiveness of the attractors of the system, there exist $X, Y, Z$, and $W$ such that $\left|x_{m}\right| \leq X,\left|y_{m}\right| \leq Y,\left|z_{m}\right| \leq Z$, and $\left|w_{m}\right| \leq W$. It also assumes that $\left|x_{s}\right| \leq X,\left|y_{s}\right| \leq Y,\left|z_{s}\right| \leq$ $Z$, and $\left|w_{s}\right| \leq W$. Thus, the error system (24) can be written in the linear interval system form (9) as

$$
D^{\alpha} \mathscr{E}=\breve{A} \mathscr{E}-B \mathscr{E},
$$

where $\mathscr{E}=\left[\begin{array}{llll}e_{1} & e_{2} & e_{3} & e_{4}\end{array}\right]^{T}, B=\operatorname{diag}\left(\beta_{1}, \beta_{2}, \beta_{3}, \beta_{4}\right)$, and the lower and upper boundaries of $\breve{A}$ are

$$
\begin{aligned}
A^{l} & =\left[\begin{array}{cccc}
-a-\sigma_{a} & a-\sigma_{a} & 0 & 1 \\
c-\sigma_{c}-Z & -1 & -X & 0 \\
-Y & -X & -b-\sigma_{b} & 0 \\
0 & -Z & -Y & r-\sigma_{r}
\end{array}\right], \\
A^{u} & =\left[\begin{array}{cccc}
-a+\sigma_{a} & a+\sigma_{a} & 0 & 1 \\
c+\sigma_{c}+Z & -1 & X & 0 \\
Y & X & -b+\sigma_{b} & 0 \\
0 & Z & Y & r+\sigma_{r}
\end{array}\right] .
\end{aligned}
$$

Assuming that the LMI condition in Theorem 2 is fulfilled, the asymptotic convergence of the synchronization errors is guaranteed. Therefore, the slave system is asymptotically synchronized with the master system.

\section{Numerical Studies}

The nominal parameters of the systems are assigned as $a=10$, $b=8 / 3, c=28$, and $r=-1$. The uncertainty bounds are assumed as $\sigma_{a}=2, \sigma_{b}=16 / 30, \sigma_{c}=5.6$, and $\sigma_{r}=0.2$ (i.e., $20 \%$ of the nominal values). The fractional order is $\alpha=0.98$. The values of $X, Y, Z$, and $W$ estimated through simulations are found to be $30,30,50$, and 250 , respectively. By solving
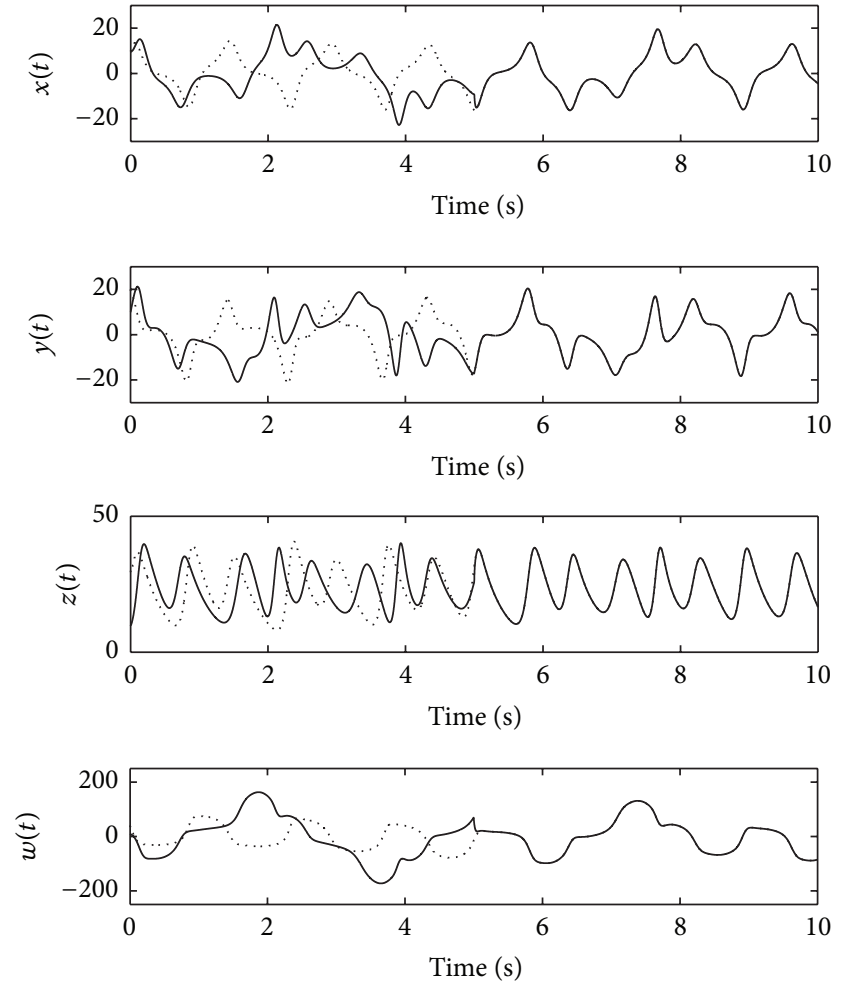

FIGURE 2: Synchronization of the hyperchaotic Lorenz systems with the nominal parameters (solid line represents slave system, dotted line represents master system).

LMI condition (10) of the error system (25), the following solution is obtained: 

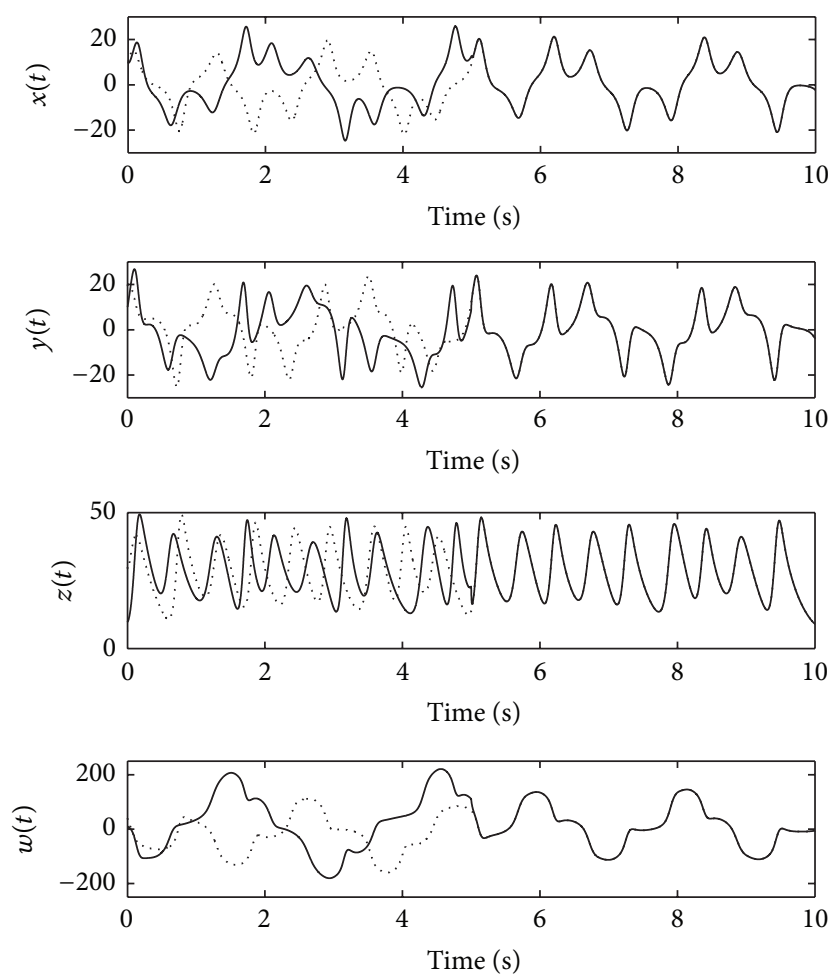

FIGURE 3: Synchronization of the hyperchaotic Lorenz systems with all parameters increased by $20 \%$ (solid line represents slave system, dotted line represents master system).
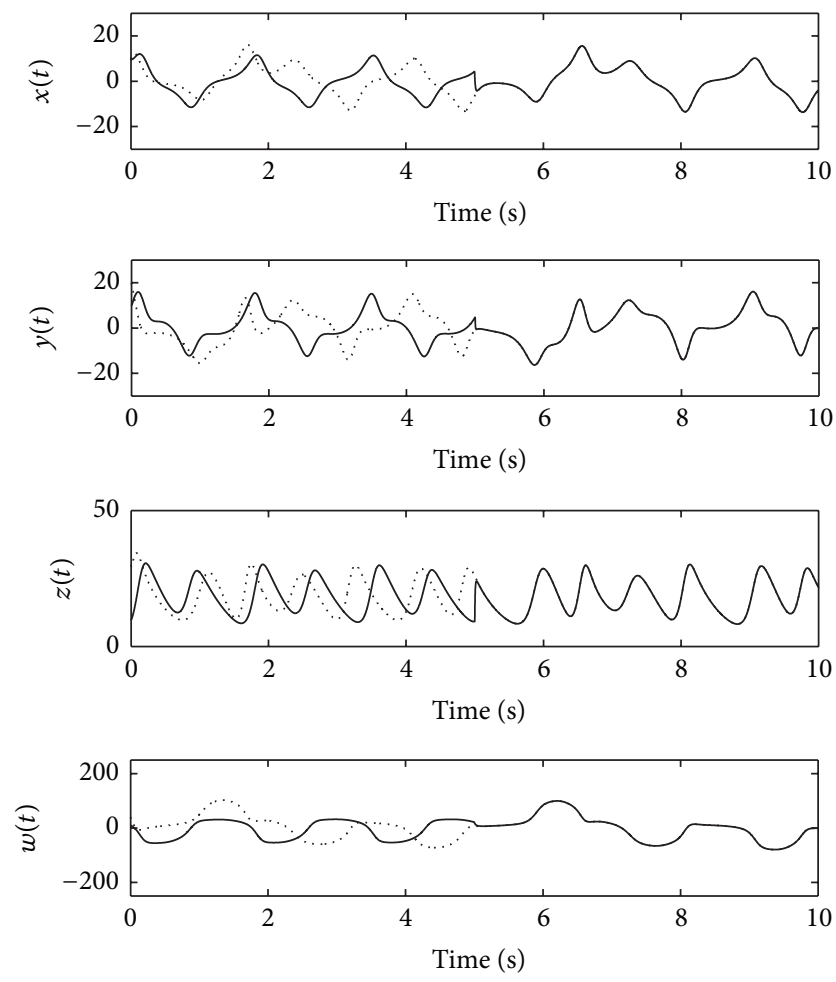

FIGURE 4: Synchronization of the hyperchaotic Lorenz systems with all parameters decreased by $20 \%$ (solid line represents slave system, dotted line represents master system).

$$
\begin{gathered}
Q=\operatorname{diag}\left(3.71 \times 10^{6}, 3.70 \times 10^{6}, 3.71 \times 10^{6}, 1.16 \times 10^{7}\right), \\
\Lambda=\operatorname{diag}\left(2.68 \times 10^{8}, 6.98 \times 10^{8}, 4.64 \times 10^{8}, 4.73 \times 10^{8}\right), \\
\alpha_{1}=7.42 \times 10^{6}, \quad \alpha_{2}=4.41 \times 10^{6}, \\
\alpha_{3}=7.42 \times 10^{6}, \quad \alpha_{4}=4.41 \times 10^{6} .
\end{gathered}
$$

This yields the following coupling parameters:

$$
\begin{array}{ll}
\beta_{1}=72.33, & \beta_{2}=188.64, \\
\beta_{3}=124.94, & \beta_{4}=40.79 .
\end{array}
$$

The state responses of the nominal master and slave systems are shown in Figure 2. Note that the controller is activated at time $=5 \mathrm{sec}$. The numerical method used in all simulations is an Adams-type predictor-corrector method. The reader is referred to [17] for the details.

Moreover, the tests with changes of the parameters are conducted. The state responses of the master and slave systems obtained after increasing all parameters by $20 \%$ are shown in Figure 3 and the ones obtained after decreasing by $20 \%$ are shown in Figure 4. In all cases, the linearly unidirectional coupling control law can effectively synchronize the slave system to the master system as desired.

\section{Conclusions}

This paper has presented a method to achieve synchronization of unidirectional coupled uncertain fractional-order hyperchaotic systems. The coupling parameters are obtained by solving an LMI condition, which is straightforward to achieve. Thanks to the existence of an LMI solution, the convergence of the synchronization errors is secured. The method has been successfully applied to the uncertain fractional-order hyperchaotic Lorenz system to illustrate the effectiveness.

\section{References}

[1] L. M. Pecora and T. L. Carroll, "Synchronization in chaotic systems," Physical Review Letters, vol. 64, no. 8, pp. 821-824, 1990.

[2] I. Podlubny, Fractional Differential Equations, Academic Press, New York, NY, USA, 1999.

[3] T. T. Hartley, C. F. Lorenzo, and H. K. Qammer, "Chaos on a fractional Chua's system," IEEE Transactions on Circuits and Systems I Fundamental Theory and Applications, vol. 42, pp. 485-490, 1995.

[4] C. Li and G. Chen, "Chaos in the fractional order Chen system and its control," Chaos, Solitons and Fractals, vol. 22, no. 3, pp. 549-554, 2004.

[5] X. Y. Wang and J. M. Song, "Synchronization of the fractional order hyperchaos Lorenz systems with activation feedback control," Communications in Nonlinear Science and Numerical Simulation, vol. 14, no. 8, pp. 3351-3357, 2009.

[6] X. Wu and Y. Lu, "Generalized projective synchronization of the fractional-order Chen hyperchaotic system," Nonlinear Dynamics, vol. 57, no. 1-2, pp. 25-35, 2009. 
[7] S. Bhalekar and V. Daftardar-Gejji, "Synchronization of different fractional order chaotic systems using active control," Communications in Nonlinear Science and Numerical Simulation, vol. 15, no. 11, pp. 3536-3546, 2010.

[8] L. Pan, W. Zhou, L. Zhou, and K. Sun, "Chaos synchronization between two different fractional-order hyperchaotic systems," Communications in Nonlinear Science and Numerical Simulation, vol. 16, no. 6, pp. 2628-2640, 2011.

[9] S. H. Hosseinnia, R. Ghaderi, A. Ranjbar N., M. Mahmoudian, and S. Momani, "Sliding mode synchronization of an uncertain fractional order chaotic system," Computers and Mathematics with Applications, vol. 59, no. 5, pp. 1637-1643, 2010.

[10] C. Yina, S. Zhonga, and W. Chen, "Design of sliding mode controller for a class of fractional-order chaotic systems," Communications in Nonlinear Science and Numerical Simulation, vol. 17, pp. 356-366, 2012.

[11] L. Chen, Y. Chai, and R. Wu, "Linear matrix inequality criteria for robust synchronization of uncertain fractional-order chaotic systems," Chaos, vol. 21, no. 4, Article ID 043107, 2011.

[12] S. Kuntanapreeda, "Robust synchronization of fractional-order unified chaotic systems via linear control," Computers and Mathematics with Applications, vol. 63, no. 1, pp. 183-190, 2012.

[13] R. Zhang and S. Yang, "Adaptive synchronization of fractionalorder chaotic systems via a single driving variable," Nonlinear Dynamics, vol. 66, no. 4, pp. 831-837, 2011.

[14] P. Zhou and W. Zhu, "Function projective synchronization for fractional-order chaotic systems," Nonlinear Analysis-Real World Applications, vol. 12, no. 2, pp. 811-816, 2011.

[15] P. Zhou, R. Ding, and Y. X. Cao, "Multi drive-one response synchronization for fractional-order chaotic systems," Nonlinear Dynamic, vol. 70, pp. 1263-1271, 2012.

[16] J.-G. Lu and Y.-Q. Chen, "Robust stability and stabilization of fractional-order interval systems with the fractional order $\alpha$ The $0<\alpha<1$ case," IEEE Transactions on Automatic Control, vol. 55, no. 1, pp. 152-158, 2010.

[17] K. Diethelm, N. J. Ford, and A. D. Freed, "A predictor-corrector approach for the numerical solution of fractional differential equations," Nonlinear Dynamics, vol. 29, no. 1-4, pp. 3-22, 2002. 


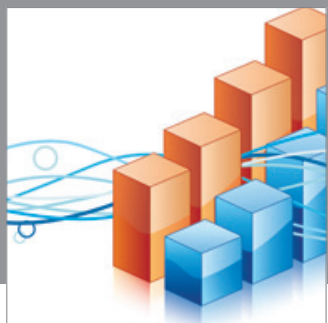

Advances in

Operations Research

mansans

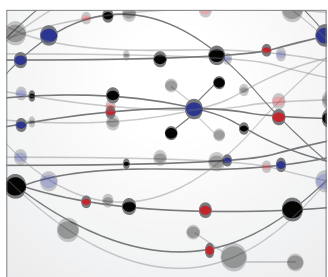

The Scientific World Journal
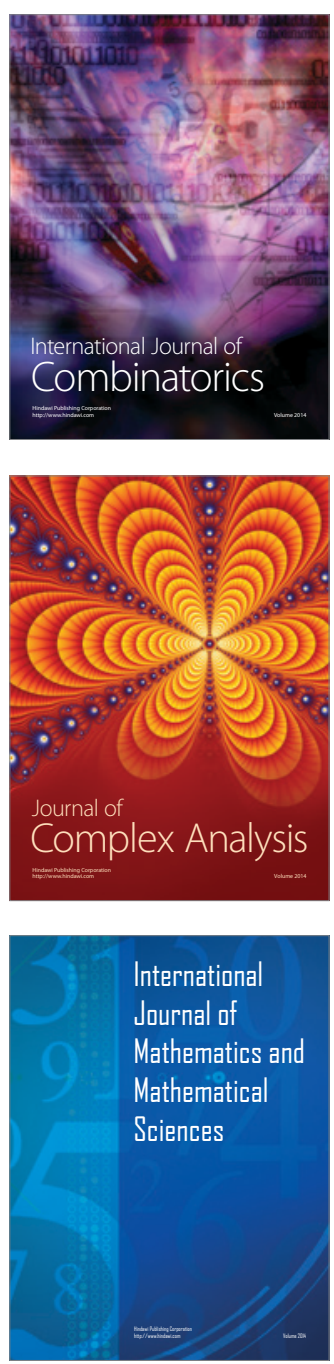
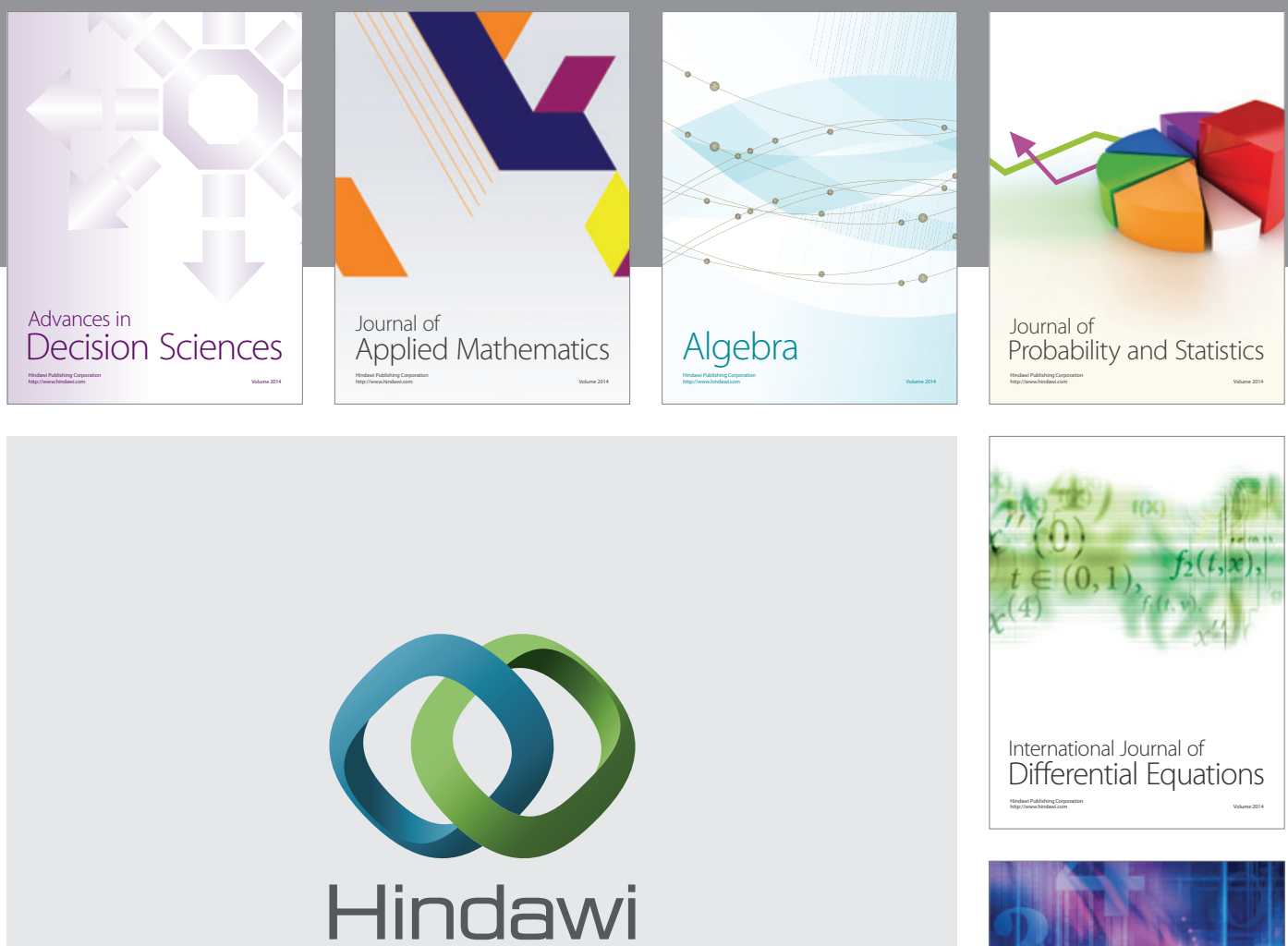

Submit your manuscripts at http://www.hindawi.com
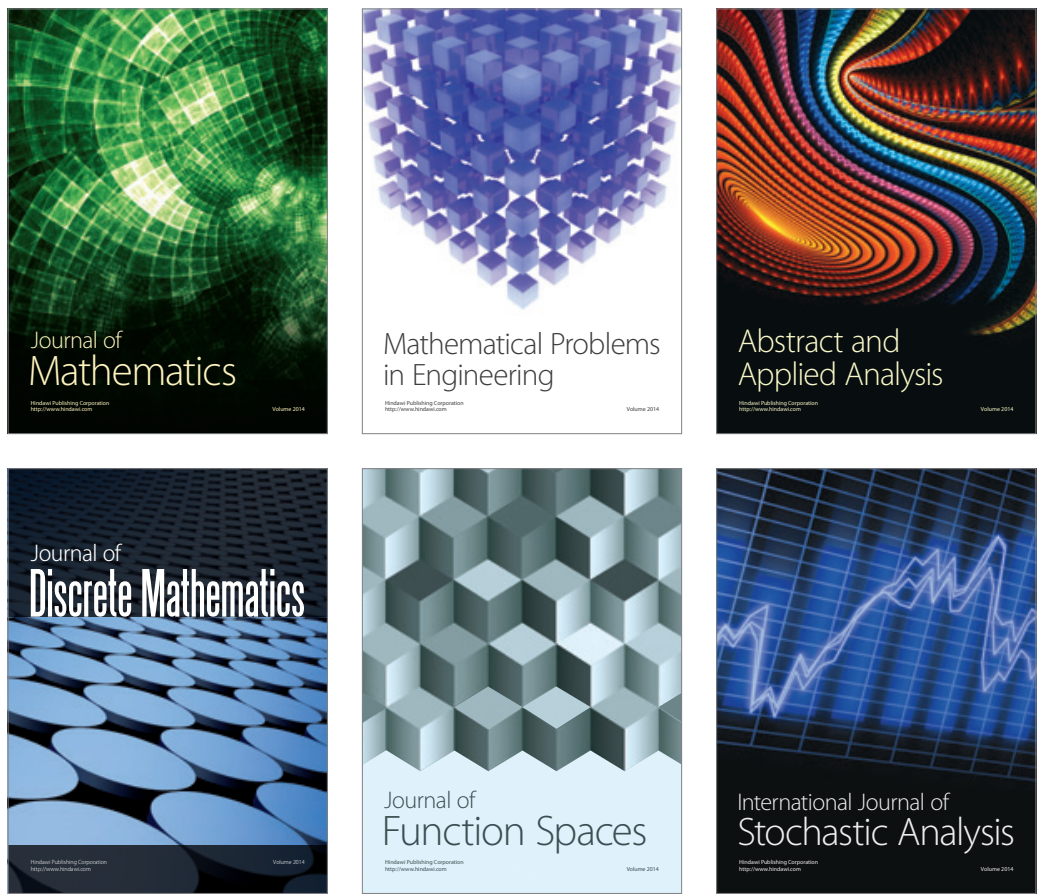

Journal of

Function Spaces

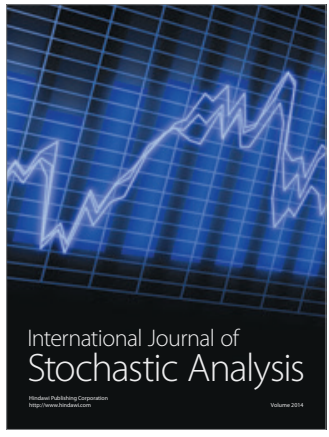

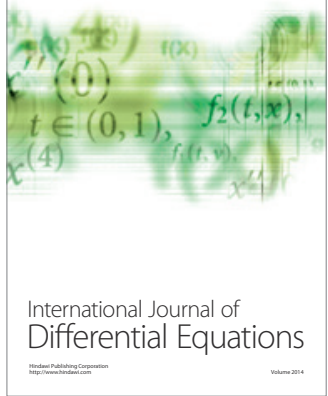
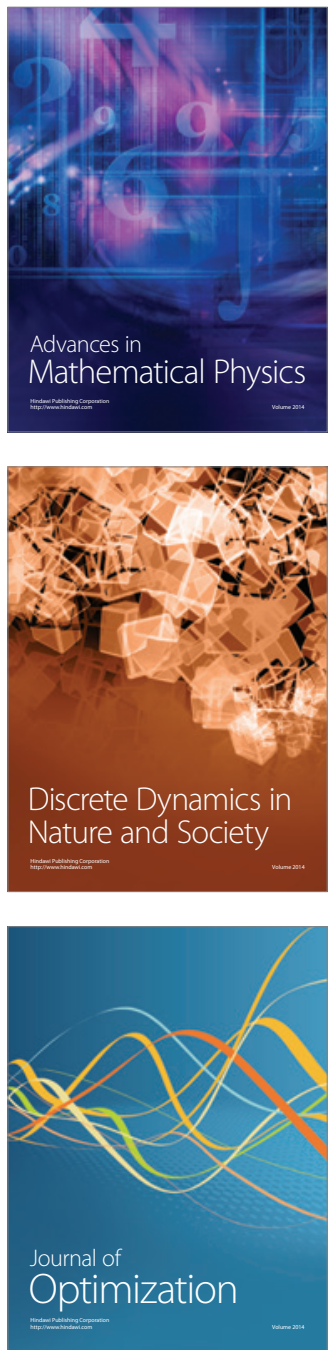